\title{
Neural noise distorts perceived motion: the special case of the freezing illusion and the Pavard and Berthoz effect
}

\author{
A. H. Wertheim · G. Reymond
}

Received: 11 May 2006/Accepted: 18 January 2007/Published online: 27 February 2007

(C) Springer-Verlag 2007

\begin{abstract}
When a slowly moving pattern is presented on a monitor which itself is moved, the pattern appears to freeze on the screen (Mesland and Wertheim in Vis Res 36(20):3325-3328, 1996) even if we move our head with the monitor, as with a head mounted display (Pavard and Berthoz in Perception 6:529-540, 1977). We present a simple model of these phenomena, which states that the perceived relative velocity between two stimuli (the pattern and the moving monitor) is proportional to the difference between the perceived velocities of these stimuli in space, minus a noise factor. The latter reflects the intrinsic noise in the neural signals that encode retinal image velocities. With noise levels derived from the literature the model fits empirical data well and also predicts strong distortions of visually perceived motion during vestibular stimulation, thus explaining both illusions as resulting from the same mechanism.
\end{abstract}

When an observer, who watches a looming pattern in a head mounted display (HMD), is abruptly moved forward (e.g. pushed on a sled), the pattern appears to suddenly become stationary on its display inside the HMD (Pavard and Berthoz 1977). The illusion is asymmetric. When pattern and observer move in opposite directions, it is very strong, and the pattern can only be seen to move if its velocity is considerably increased, although it is then severely underestimated. When pattern and sled move in the

\footnotetext{
A. H. Wertheim ( $\square)$

Department of Psychonomics,

Utrecht University, Utrecht, The Netherlands

e-mail: a.wertheim@fss.uu.nl

G. Reymond

Renault, Technical Center for Simulation,

Technocentre TCR AVA 013, Guyancourt, France
}

same direction the threshold cannot be measured properly, as subjects find their percepts ambiguous and difficult to report. The phenomenon looks like a confusion between egocentric and exocentric frames of reference, because when the pattern moves in the direction opposite to that in which the observer is pushed, it actually approaches stationarity relative to external space. We will henceforth refer to this phenomenon as the P\&B-effect.

A somewhat similar illusion, not involving vestibular stimulation, occurs when we watch a visual pattern scrolling slowly at a fixed velocity across a monitor. As soon as the monitor itself is moved, the pattern appears to stop moving on the monitor (Mesland and Wertheim 1996). We will call this the freezing illusion. Just as the P\&Beffect, the illusion is asymmetric, being most pronounced when monitor and pattern move in opposite directions (i.e. when the pattern approaches stationarity relative to external space). And beyond that elevated threshold, pattern velocity is severely underestimated. Recently, the same phenomenon has also been reported with rotating stimuli which appear to stop rotating when the monitor is rotated itself, or when the rotating stimulus is superimposed on a rotating background (Duersteler 2005).

Mesland and Wertheim quantified the freezing illusion with a monitor placed on a sled which moved (at $40 \mathrm{~cm} / \mathrm{s}$ ) alongside a subject who was seated next to the sled, looking in forward direction parallel to the tracks of the sled while keeping the eyes fixed on a fixation mark placed a few meters straight ahead (see Fig. 1). On the sled the monitor screen faced sideward, towards the left side of the subject and was made visible for only $0.5 \mathrm{~s}$. Thus the monitor and the pattern (a vertical grating) moving horizontally across it, were perceived peripherally, while the sled moved either in forward direction (away from the subject) or in backward direction (towards the subject). 


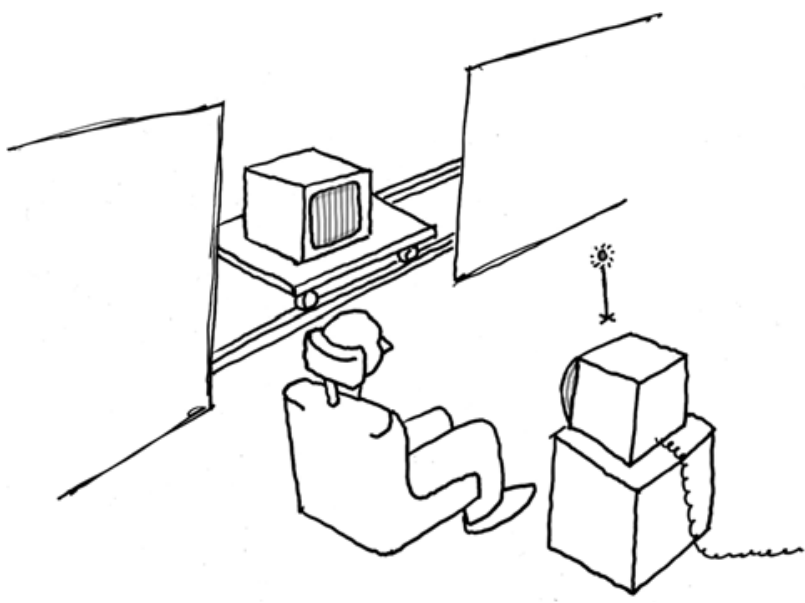

Fig. 1 Sketch of the experimental setup of the Mesland and Wertheim (1996) study on the freezing illusion

The perceived velocity of the grating on the moving monitor was measured by matching the (adjustable) velocity of another grating to it. The other grating was also presented peripherally for $0.5 \mathrm{~s}$, but on a stationary monitor placed to the right of the subject. Figure 2 gives the thus estimated grating velocities on the moving monitor.

As can be seen from the lower scale, when grating and monitor physically moved in opposite directions, the threshold for seeing the grating movement on the monitor was very high. It extended even to the point where the grating was actually stationary in space (where grating velocity on the monitor and monitor velocity in space are equal but opposite in direction). Beyond that threshold, grating velocity was strongly underestimated. However, when grating and monitor moved physically in the same direction the threshold was much smaller and beyond it grating velocity on the monitor appeared to be more or less unbiased.

On the other hand, when considering the data from a retinal perspective (upper scale), the situation was different: here the high threshold occurred when the images of grating and monitor moved in the same direction across the retinae, and beyond that threshold grating velocity was perceived more or less veridically. When the images of grating and monitor moved in opposite directions across the retinae, there was barely a threshold, but beyond it grating velocity was strongly underestimated.

At the time of their publication, the freezing illusion and the P\&B-effect could not be explained. However, presently we propose a quite simple explanatory model for both phenomena, based on the effects of neural noise in combination with a well-known general principle of motion perception.

This principle is that the perceived velocity of a stimulus relative to external space corresponds to the extent its retinal image velocity cannot be explained by movements
Fig. 2 Perceived grating velocity across the moving monitor ( $Y$-axis) as a function of its physical velocity (lower $X$ axis). The arrows below the graph illustrate the velocity and direction in which the grating moved on the moving monitor (rectangles) which itself moved at $40 \mathrm{~cm} / \mathrm{s}$ backward, i.e. towards the subject (left panel), or forward, i.e. away from the subject (right panel). The upper velocity scale gives the retinal image velocity of the grating, which across a large range is proportional but opposite in sign to physical grating velocity in space (because the eyes remained fixed in space). This upper scale thus allows for evaluating perceived grating velocity on the monitor as a function of grating retinal image velocity. So here the rectangles and the arrows refer to the retinal directions and velocities of the images of the monitor and of the grating on the monitor. (Adapted from Mesland and Wertheim 1996)

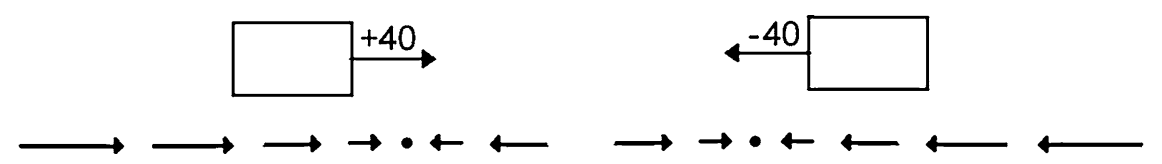

Retinal grating velocity

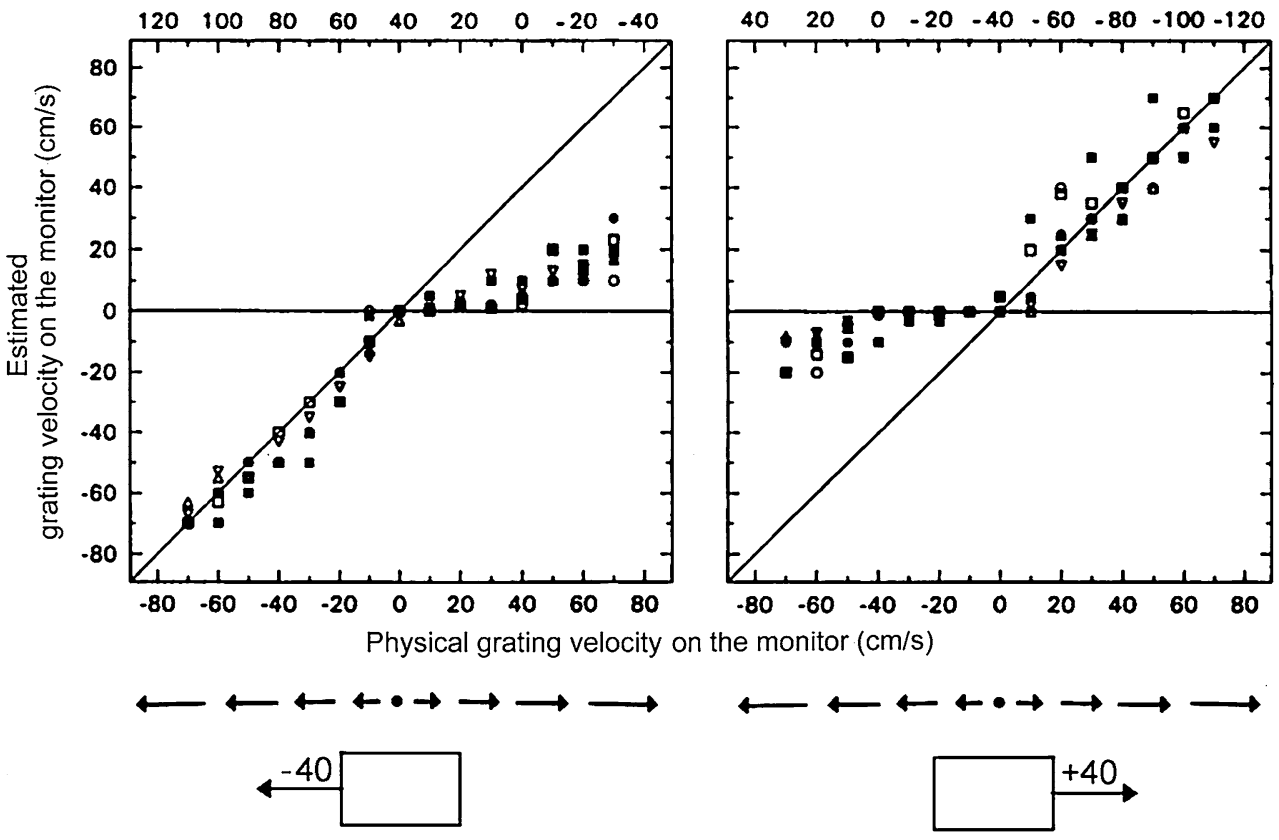


of the eyes (i.e. the retinae) in space. Stated in vectorial terms, this means that perceived stimulus velocity in space corresponds to a difference vector, expressed as the vector which represents the velocity of the eyes in space minus the vector that represents stimulus retinal image velocity.

When eye movements are made while the head remains stationary in space, the vector representing eye velocity in space is generally assumed to stem from an 'efference copy', which is a signal that encodes ocular velocity in the head (Von Holst and Mittelstaedt 1950; Mittelstaedt 1990), presumably deriving from the neural commands to the ocular musculature. But during head or ego-movements, eye movements in the head do not reflect how the eyes move in space. Thus it has been proposed that the more general case is that ocular velocity in space is conveyed to the visual system by a compound signal that consists of an efference copy plus an ego-motion signal (most likely deriving from vestibular reactivity, neck muscle afferents etc). This compound signal has been termed 'reference signal' (see Wertheim 1994, for details).

Thus we may assume that an object is perceived to move in external space with a velocity that corresponds to the difference between this reference signal and the retinal stimulus image velocity signal. However, as shown elsewhere (Wertheim 1981; Wertheim and Van Gelder 1990; Wertheim 1994), perceived stimulus velocity in space actually corresponds to this difference minus the Just Noticeable Difference (JND) between the two signals. This JND can be interpreted as the combined intrinsic neural noise within the two signals. As long as the difference between the two signals is less than this noise level (i.e. remains smaller than this JND in absolute terms), perceived stimulus velocity in space remains zero, i.e. such stimulus motion is not perceived. Thus psychophysically speaking, the threshold for perceiving motion in space corresponds to that JND. Accordingly we can state the basic principle of motion perception as follows: The above threshold perceived velocity in space of any particular stimulus always corresponds to the difference between a reference signal and the concurrent retinal stimulus image velocity signal minus the JND between them, and stimulus stationarity in space is perceived as long as the difference between the two signals remains smaller than that JND in absolute terms.

Now consider the freezing illusion. According to the basic principle, the perceived velocity of the monitor in space $\left(V_{\text {perc }_{\text {mon/space }}}\right)$ corresponds to the difference between the reference signal $\left(V_{\text {ref }}\right)$ and the velocity of the monitor image across the retinae $\left(V_{\mathrm{mon} / \mathrm{ret}}\right)$ minus a JND, or:

$V_{\text {perc }_{\text {mon } / \text { space }}}=V_{\text {ref }}-V_{\text {mon/ret }}-\mathrm{JND}_{(1)}$.

And this formula is restricted by the definition of the threshold for perceiving the monitor as moving in space: If the absolute value of ( $\left.V_{\text {ref }}-V_{\text {mon/ret }}\right)$ is less than or equal to the absolute value of $\mathrm{JND}_{(1)}$, monitor motion in space remains below threshold and is not perceived, i.e. $V_{\text {perc }}$ mon/space remains zero.

Similarly, the perceived velocity of the grating in space $\left(V_{\text {perc }_{\text {grat } / \text { space }}}\right)$ corresponds to the reference signal $\left(V_{\text {ref }}\right)$ minus the velocity of the grating image across the retinae (Vgrat/ret) minus a JND, or:

$V_{\text {perc }_{\text {grat } / \text { space }}}=V_{\text {ref }}-V_{\text {grat } / \text { ret }}-\mathrm{JND}_{(2)}$.

And here too the threshold restriction applies: as long as the absolute value of $\left(V_{\text {ref }}-V_{\text {grat/ret }}\right)$ remains smaller than or equal to the absolute value of the $\mathrm{JND}_{(2)}, V_{\text {perc }}$ grat/space remains zero.

These two formulas thus describe the two separate percepts of monitor motion and grating motion relative to external space. Our explanation of the freezing illusion consists simply of subtracting these two perceived velocities. The perceived velocity of the grating relative to the monitor $\left(V_{\text {perc }_{\text {grat } / \text { mon }}}\right)$ then becomes:

$V_{\text {perc }_{\text {grat } / \text { mon }}}=V_{\text {perc }_{\text {grat/space }}}-V_{\text {perc }_{\text {mon/space }}}-\operatorname{JND}_{(3)}$.

This $\mathrm{JND}_{(3)}$ of course reflects another noise component causing a threshold restriction: $V_{\text {perc }}$ grat $m o n_{\text {mon }}$ remains zero (below threshold) as long as the absolute value of the factor $\left(V_{\text {perc }_{\text {grat } / \text { space }}}-V_{\text {perc }_{\text {mon/space }}}\right)$ remains smaller than or equal to the absolute value of this $\mathrm{JND}_{(3)}$.

We can measure $V_{\text {ref }}, V_{\text {mon/ret }}$ and $V_{\text {grat/ret }}$ (from stimulus, eye and head velocity recordings). We can then use formula's (1) and (2) to calculate $V_{\text {perc }_{\text {rat } / \text { space }}}-$

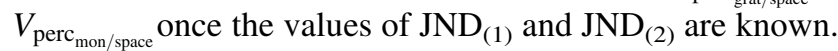
From experiments in which thresholds for stimulus motion are obtained during pursuit eye movements (made to a fixation point sweeping across a stimulus), the combined neural noise between a retinal and a reference signal can be estimated as approximately $8 \%$ of signal magnitude (see e.g. Wertheim 1981, 1994). The difference of two uncorrelated Gaussian noisy signals is Gaussian itself, with a variance equal to the sum of the two signals' variances. Assuming such independence, and setting the JND level to one standard deviation, we may thus calculate $\mathrm{JND}_{(1)}$ and $\mathrm{JND}_{(2)}$ in Eqs. 1 and 2 as $8 \%$ of the square root of the sum of the squares of the two noise signals involved. (But since it is known that with briefly visible stimuli thresholds for motion are higher, these noise levels could still be somewhat higher). However, this causes the JND to be always positive, even though it should have the same sign as the sensory (retinal) stimulus velocity signal. So in the case of formulas (1) and (2) the JND should be given the sign opposite to $V_{\text {perc }_{\text {mon/space }}}$ and $V_{\text {perc }_{\text {grat/space }}}$, respectively. What 
remains to be done is to estimate the $\mathrm{JND}_{(3)}$ in Eq. 3 and then we can calculate $V_{\text {perc }}$ rat/mon. The question thus becomes: what could be that estimate?

As mentioned above, a JND can be thought of as representing intrinsic noise of the neural signals involved. In the Mesland and Wertheim study the freezing illusion was perceived without head or eye movements. Hence we may assume that the illusion stemmed only from retinal information, i.e. from retinal motion (velocity) signals. What then are these signals? We propose that basically there are two retinal velocity signals involved, one referring to absolute motion and one to relative motion. The first encodes the retinal velocity of the monitor image $\left(V_{\text {mon/ret }}\right)$. The second encodes the relative velocity between the image of the moving monitor and that of the grating inside it $\left(V_{\text {delta }}\right)$, i.e. the retinal projection of the difference between monitor velocity and grating velocity on the monitor. Having thus defined the two relevant retinal motion signals, we must now estimate the noise intrinsic in them.

From an experiment in which subjects moved their eyes across two briefly visible patterns moving concurrently at slightly different velocities, velocity discrimination thresholds-for perceiving a velocity difference between the two patterns-were on average as high as $35 \%$ of retinal image velocity (Wertheim and Niessen 1986; Wertheim 1994). Hence we may take this as the JND between our two retinal velocity signals, i.e. we may estimate
$\mathrm{JND}_{(3)}$ in Eq. 3 as $35 \%$ of the square root of the sum of the squares of $\left(V_{\text {mon/ret }}\right)$ and $\left(V_{\text {delta }}\right)$. Its sign is assumed to be that of the main retinal signal defined by the monitor, i.e. opposite to that of $V_{\text {perc }_{\text {mon/space }}}$. With this estimate we can calculate $V_{\text {perc } \text { grat } / \text { mon }}$ in Eq. 3 .

To test our explanatory model of the freezing illusion we used the data from a more recent replication of the original Mesland and Wertheim study. Method, apparatus and procedures were identical to those of the original study, using also three subjects and two replications per subject. The individual results which illustrate data variability are given in Fig. 3. We then used the above reasoning to calculate the predicted values of $V_{\text {perc }_{\text {grat }} \text { mon }}$. Finally, since the perception of motion is affected when stimuli are presented peripherally and only briefly, a general gain factor $(G)$ was applied. When this gain factor was set to 0.80 this yielded the dotted lines in Fig. 4. The model simulations were computed using Matlab ${ }^{\circledR}$ (The Matworks, Inc). The appendix describes the model in standard mathematical terms.

The data replicated very well in both experiments (compare Figs. 2 and 3). The goodness of fit of the model is very high: for the $n=15$ data points in each panel of Fig. 4, the Pearson product-moment correlation coefficient is $r>0.99$. The non-linearity in the model curve stems from the fact that the $\mathrm{JND}_{(3)}$ factor is not linear, being (35\% of) the square root of the sum of the squares of $V_{\text {mon/ret }}$
Fig. 3 Individual data from the replication experiment. See legend of Fig. 2 for details

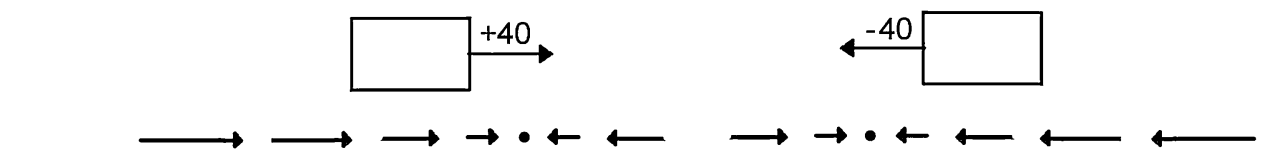

Retinal grating velocity

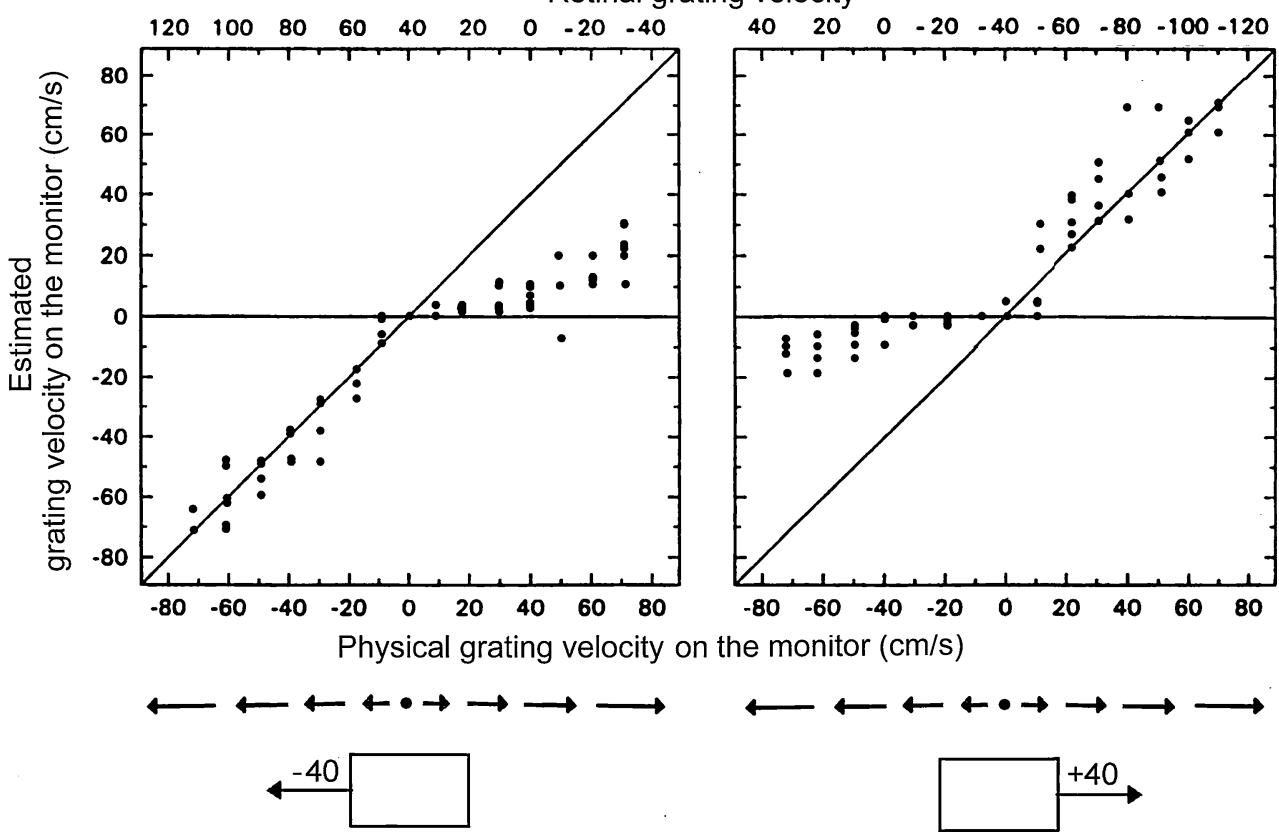


and $V_{\text {delta.ret }}$. However, the model response becomes almost linear at high stimulus velocity values.

Another striking aspect is the discontinuity which occurs just beyond the threshold when grating and monitor move in the same direction. To understand this, consider the case where the sled moves forward, i.e. $V_{\text {perc }}$ mon/space is positive. Since grating and monitor move in the same positive direction and the grating moves faster in space than the monitor, the factor $\left(V_{\text {perc }_{\text {grat } / \text { space }}}-V_{\text {perc }_{\text {mon/space }}}\right)$ in formula (3) is positive as well. As long as its absolute value remains less than the absolute value of $\mathrm{JND}_{(3)}, V_{\text {perc }_{\text {grat } / \text { mon }}}$ remains zero, and we will not see the grating move on the monitor. If we increase grating velocity on the monitor, the value of the $\left(V_{\text {perc }_{\text {grat/space }}}-V_{\text {perc }_{\text {mon/space }}}\right)$ factor increases, and the threshold will be reached when its absolute value reaches the absolute value of $\mathrm{JND}_{(3)}$. However, the value of the $\mathrm{JND}_{(3)}$ is actually negative, because, as mentioned earlier, its sign is opposite to that of $V_{\text {perc }_{\text {mon/space }}}$.Hence, when crossing the threshold, $V_{\text {perc }_{\text {grat } m o n}}$ suddenly jumps from zero to the value of $G \times 2 \times \mathrm{JND}_{(3)}$. (See lower scale of Fig. 4, where this discontinuity happens at +18 , right panel, and at -18 , left panel, where the sled moved in the other direction inverting the signs). Consequently, just above this threshold slow velocities can simply not be perceived, and higher stimulus velocities are perceived with little bias.
Note that this does not happen when the monitor moves forward but the grating moves backward on the monitor. The JND then remains negative, but the sign of the factor $\left(V_{\text {perc }_{\text {grat } / \text { space }}}-V_{\text {perc }_{\text {mon/space }}}\right)$ is now negative. Once that factor becomes equal to $\mathrm{JND}_{(3)}$ in an absolute sense, the threshold is reached. So when the threshold is crossed

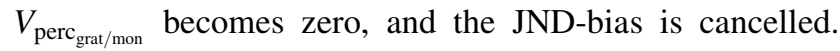
From then on $V_{\text {perc }}$ grat/mon just increases from zero.

Actually, this reasoning is not new. It was used earlier (Wertheim 1994, pp. 301) to explain a somewhat similar finding, reported by Wertheim and Van Gelder (1990). They used a matching paradigm to measure perceived above threshold stimulus velocity during a pursuit eye movement (made to a fixation point sweeping across the stimulus). Motion thresholds were elevated in proportion to eye velocity (which was expected, because, according to Weber's law, larger reference signals have higher JND's, i.e. noise levels, a JND being a fixed fraction-Weberfraction - of signal magnitude). The results showed that above threshold stimulus velocity for stimuli moving in the same direction as the eyes was underestimated in proportion to that elevated threshold, but no such underestimation occurred when stimulus and eyes moved in opposite directions. Assuming that perceived stimulus velocity in space equals $V_{\text {ref }}-V_{\text {stim/ret }}-\mathrm{JND}$, Wertheim (1994) explained this asymmetry (at least partially) with the same
Fig. 4 Group average data from the replication experiment. Dotted lines model predictions. See legend of Fig. 2 for details

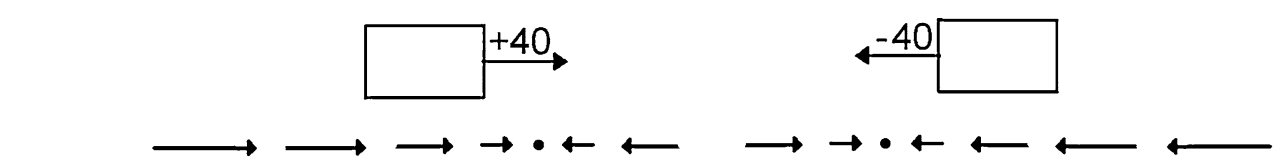

Retinal grating velocity

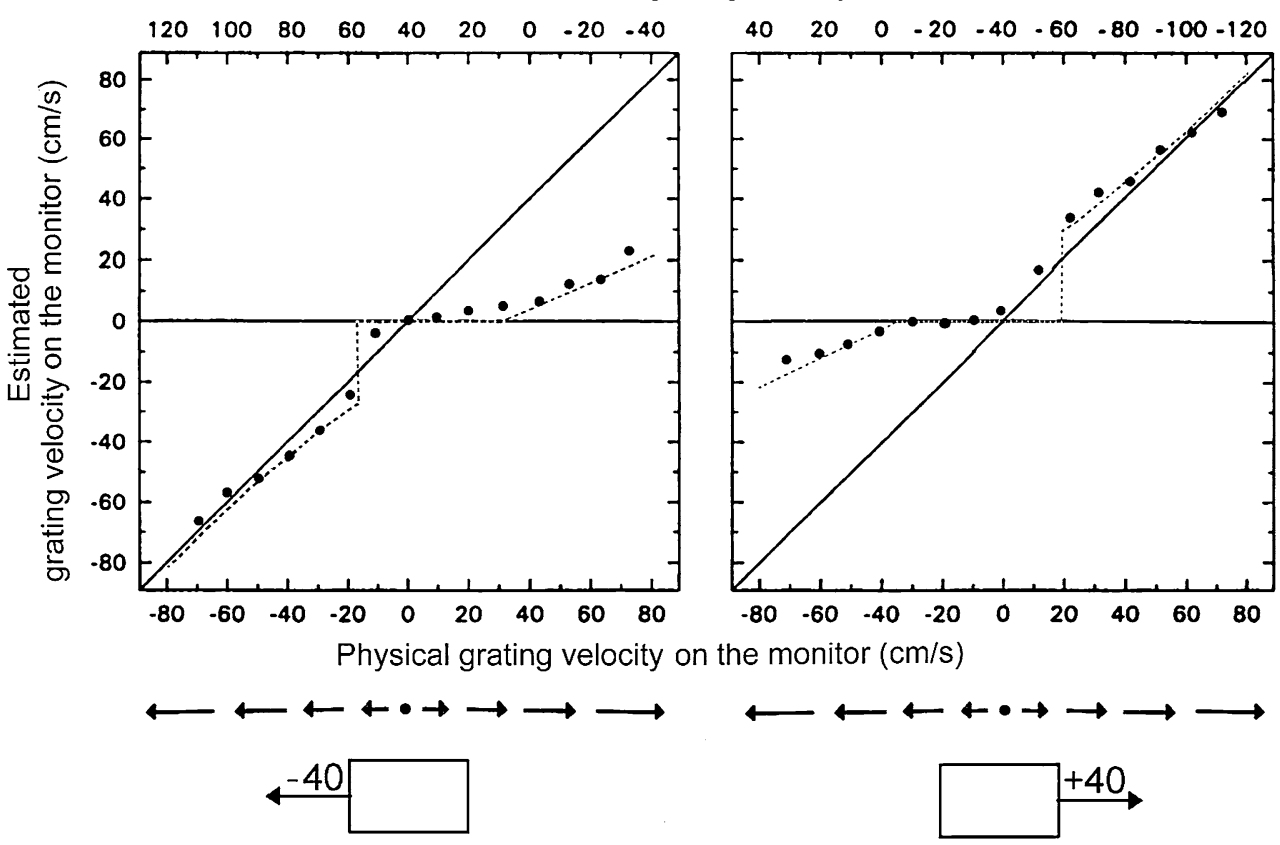


reasoning as used presently. When the eyes move across a stimulus that moves in the same direction as the eyes, the stimulus generates a retinal signal $V_{\text {stim/ret }}$ that is smaller than the eye movement induced reference signal $V_{\text {ref }}$. This causes the $\left(V_{\text {ref }}-V_{\text {stim/ret }}\right)$ factor to be positive. If it is larger than one JND, stimulus motion is perceived, although with a threshold bias: its velocity should be underestimated in proportion to the JND. However, when the stimulus moves in the direction opposite to the eyes, it generates a retinal signal larger than the reference signal, causing the $\left(V_{\text {ref }}-V_{\text {stim/ret }}\right)$ factor to be negative. As long as it is absolute value is less than the absolute value of one JND no motion is perceived. But above this threshold, more or less veridical motion is perceived, because the threshold bias is now counteracted by a $-2 \times$ JND velocity jump.

An important consequence of the present explanation of the freezing illusion is that it does not matter much whether or not eye movements are made. The point is that image velocity can be defined as eye velocity in space minus stimulus velocity in space. But if we replace the retinal image velocity terms in Eqs. 1 and 2 accordingly, formula (3) changes into:

$V_{\text {perc }_{\text {grat } / \text { mon }}}=V_{\text {mon/ret }}-V_{\text {grat } / \text { ret }}-\mathrm{JND}_{(3)}$.

Hence, the eye movement components are cancelled out, and now the model is based on a comparison of just two retinal signals. However, since neural noise cannot just disappear, the noise created by the eye movements (i.e. by the eye movement induced reference signals that dropped out of the equation) must somehow still be present in the noise component of Eq. 4. However, this extra noise consists of the noise components in Eqs. 1 and 2 and these are quite small. They will only slightly affect the already quite high value of $\mathrm{JND}_{(3)}$. As a result, if we use Eq. 4 in our model, the predictions barely differ from the ones in Fig. 4. Hence it does not really matter much if one tracks the monitor or the grating with the eyes, or keeps the eyes fixed at a stationary point in the environment: the freezing illusion will always be seen.

The discontinuity mentioned above, which implies that slow above threshold velocities cannot be seen when grating and monitor move in the same direction, brings to mind the ambiguity reported to occur with the P\&B-effect when pattern and observer moved in the same direction.

And indeed we can use the same logic to explain the P\&B-effect: A pattern moving across a head mounted display creates a $V_{\text {pat/ret }}$ signal, and during head or egomotion a $V_{\text {ref }}$ signal is generated (which includes an additional efference copy component if the eyes also move in their orbits). If we substitute the HMD for the monitor in formula (1) we get the perceived velocity of the HMD in space, while formula (2) gives the perceived velocity of the pattern in external space. Thus the perceived velocity of the pattern relative to the HMD is given by formula (5):

$V_{\text {perc }_{\text {pat } / \mathrm{HMD}}}=V_{\text {perc }_{\text {pat } / \text { ppace }}}-V_{\text {perc }_{\mathrm{HMD} / \text { space }}}-\mathrm{JND}_{(4)}$.

And again there must be a threshold restriction: $V_{\text {perc }_{\text {pat/Нм }}}$ remains zero (below threshold) as long as the

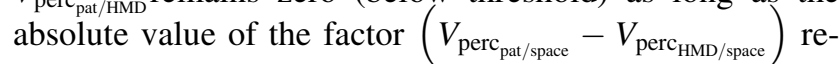
mains less than or equal to the absolute value of $\operatorname{JND}_{(4)}$. Of course the magnitude of this $\mathrm{JND}_{(4)}$ is as yet unknown, but it could be obtained from psychophysical threshold measurements.

It may be questioned whether $V_{\text {perc }}{ }_{\mathrm{HMD} / \text { space }}$ in Eq. 5 is really a percept, i.e. whether we really perceive a HMD as moving in space when we move our head. But that depends on one's definition of the term "perceive". However, this issue is not really relevant. The point is that for $V_{\text {perc }}{ }_{\mathrm{HMD} / \text { space }}$ in formula (5), we may substitute perceived ego-velocity in space, because these two percepts should be more or less equal. This means that the P\&B-phenomenon can also be understood as the resulting from a subtraction of two percepts (a pattern motion percept and an ego motion percept), minus a noise component, just as the freezing illusion. Therefore, it should exhibit the same dynamics as the freezing illusion. Thus the P\&B-phenomenon should also show a just above threshold discontinuity when pattern and HMD (i.e. the head) move in the same direction. This nicely explains why this threshold could not properly be measured: subjects experienced a perceptual ambiguity around that threshold because slow pattern motion simply cannot be perceived. One either sees the pattern move relatively fast across the HMD screen, or not at all.

Note also that in both cases a similar perceptual paradox occurs: in the freezing illusion experiments, when the pattern moved at $40 \mathrm{~cm} / \mathrm{s}$ in the direction opposite to the monitor, it was stationary in space. But at the same time was seen as stationary relative to the moving monitor. Similarly, in case of the P\&B-effect the pattern was seen as stationary relative to the moving observer while it was stationary in space as well. No wonder Pavard and Berthoz reported that it looked like a confusion of frames of reference. The present model just explains these paradoxes as caused by the high noise level of the neural signals involved.

Our reasoning illustrates an important theoretical issue: the intrinsic neural noise within our perceptual system is a factor that must be taken into account in models concerning visual percepts of velocity and motion thresholds, because it may cause considerable distortions of such percepts. The freezing illusion and the P\&B-effect are just two examples.

There are many other phenomena that are caused by such intrinsic noise as well. For example, slightly different 
retinal velocity signals are created by the leaves on a tree. When looking at the tree from a moving car, all retinal velocities increase, but the small differences between them remain the same. Since large retinal velocity signals are intrinsically more noisy (according to Weber's law), those small velocity differences will now remain below threshold. Hence from a moving car we cannot see the leaves move. They "freeze" on the tree. A similar motion stopping effect is easily seen when we shake our head in front of a slowly moving stimulus pattern (e.g. a scrolling text on our TV, or a looming pattern on a fronto-parallel screen). This reasoning has also been used (Nakayama 1981) to predict that the kinetic depth percept disappears when pursuit eye movements are made to a small fixation point sweeping across the display. The point is that kinetic depth percepts too depend on the detection of small differences between retinal velocities. When eye movements increase all retinal velocity signals, the intrinsic noise levels of the retinal signals increase as well, and may become too high to detect the small differences between them (which remain unaffected by the eye movements), thus causing the kinetic depth effect to disappear. As mentioned earlier, noise between retinal and reference signals also explains why during pursuit eye movements the threshold for stimulus motion increases in proportion to eye velocity (Murphy 1978; Wertheim 1981). The Aubert-Fleishl effect (the perceived velocity of a stimulus is higher when it moves across stationary eyes than when it is pursued with the eyes) has been explained similarly as due to the increased noise in reference signals which is proportional to ocular velocity (Wertheim and Van Gelder 1990). Since reference signals generated by ego-motion (e.g. from vestibular reactivity), also have intrinsic noise proportional to their magnitude, one should also expect that during ego-motion the perceived velocity of a moving stimulus is reduced. This is indeed a well-known phenomenon (Buechele et al. 1980; Probst et al. 1986; Wallach 1987). In fact we should expect similar effects if reference signals are generated in situations where the brain is "fooled" into believing that ego-motion takes place. For example walking or running on a treadmill, or cycling on a bicycle ergometer could suggest ego motion to the brain, generating a reference signal proportional to the 'faked' ego-velocity. If so, the perceived velocity of a looming stimulus concurrently projected on a frontoparallel screen, may be underestimated in proportion to that 'faked' egovelocity. Such effects have indeed been reported (Pelah and Thurell 2001; Thurell and Pelah 2002; Durgin et al. 2005). However, it should be noted that in these cases the slowing down of perceived motion could also have stemmed from head shaking movements made during walking, running or cycling, which might create a situation similar to that of the freezing leaves on the tree as discussed above. However, in several pilot studies in which we restricted head movements, we too observed these velocity underestimating effects during 'fake' ego motion (ergometer cycling). But this occurred most notably only during the initial phases of the cycling activity. Our explanation is that only during the initial phase of such 'faked' ego-motion, a reference signal is generated, because only that phase suggests ego-acceleration (the vestibular system only reacts to accelerations). We noted that in such circumstances the looming pattern may even freeze altogether, i.e. become perceived as completely stationary on the screen.

If our reasoning is correct it has practical consequences as well: whenever noise in the perceptual system can be expected to increase (i.e. whenever neural velocity signals grow in magnitude), the perceived velocity of objects in our environment may become strongly underestimated. This is likely to happen (and may interfere with correct visuo-motor performance) during any kind of vestibular stimulation, such as during accelerations or decelerations in cars, aircraft, or simulators, or when moving the head while wearing a head mounted display. One such phenomenon is fun to demonstrate: just sit in the passenger seat of a car and watch a laptop computer with a constant low velocity scrolling pattern. Whenever the driver accelerates, decelerates, or speedily takes a strong curve, you will see the pattern suddenly freeze on the laptop. If you do not have access to a car, take the laptop with you on an office chair with wheels, and ask for a push.

Acknowledgements This research was carried out partly in the framework of the european project ULTIMATE (Eureka \#1493). We thank Prof A. Berthoz (LPPA, CNRS-College de France) and Dr. A. Kemeny (Renault, Technical Center for Simulation) for their support and helpfull scientific discussions.

\section{Appendix}

\section{Mathematical formulation of the model}

The physical stimuli that represent the model input variables are: physical velocity of monitor in space $M$; velocity of the image (grating) on the monitor $R$; and eye velocity in space $E$. The psychophysical constants of the model are: the Weber fractions for the difference between retinal and reference signals $w$, and for the difference between two retinal velocity signals $w_{\delta}$; and a stimulus-dependent gain factor $g$ for the visual perception of velocities.

Let $\Theta(x, t)$ be a function defined by: $\Theta(x, t)=0$ if $|x|<t$ and $\Theta(x, t)=x-t$ otherwise.

The perceived velocity of the monitor in space is related to the difference between the retinal velocity of the monitor $V_{\text {mon/ret }}=E-M$, and the reference signal $V_{\text {ref }}$ which is 
equal to $E$ for a still observer and to $E+V_{\text {head/space }}$ for a moving observer:

$\hat{V}_{\text {mon } / \text { space }}=\Theta\left(V_{\text {ref }}-V_{\text {mon } / \text { ret }}, \mathrm{JND}_{1}\right)$,

where

$\mathrm{JND}_{1}=-\operatorname{sign}\left(V_{\mathrm{mon} / \mathrm{ret}}\right) w \sqrt{V_{\text {ref }}^{2}+V_{\mathrm{mon} / \mathrm{ret}}^{2}}$.

The perceived velocity of the grating in space is related to the difference between the retinal velocity of the grating $V_{\text {grat/ret }} E-(M+R)$, and the reference signal $V_{\text {ref: }}$ :

$\hat{V}_{\text {grat } / \text { space }}=\Theta\left(V_{\text {ref }}-V_{\text {grat } / \text { ret }}, \mathrm{JND}_{2}\right)$,

where

$\mathrm{JND}_{2}=-\operatorname{sign}\left(V_{\text {grat } / \mathrm{ret}}\right) w \sqrt{V_{\text {ref }}^{2}+V_{\text {grat } / \text { ret }}^{2}}$

The perceived velocity of the grating relative to the monitor is related to the difference between the two perceived velocities in space, using a JND based on the difference between the two retinal signals involved. These are assumed to be: the retinal velocity of the monitor $V_{\mathrm{mon} / \mathrm{ret}}=E-M$; and the velocity difference between the image of the monitor and the grating within it $V_{\text {delta }}=-(M-R)$. Thus:

$\hat{V}_{\text {grat } / \text { mon }}=\Theta\left(\hat{V}_{\text {grat } / \text { space }}-\hat{V}_{\text {mon/space }}, \mathrm{JND}_{3}\right)$,

where

$\mathrm{JND}_{3}=-\operatorname{sign}\left(\hat{V}_{\text {mon/space }}\right) w_{\delta} \sqrt{V_{\text {mon/ret }}^{2}+V_{\text {delta }}^{2}}$.

This value is then to be factored by a stimulus-dependent gain $g$ to model the experimentally measured perceived velocity of the grating on the monitor.

Note that the model is deterministic and treats JND's as signed values, the sign of which is defined as opposite to that of the perceived velocity of the monitor or grating in space. However, since we think of a JND as intrinsic noise, it should be an interesting challenge to translate the model in more probabilistic terms.

\section{References}

Buechele W, Degner D, Brandt T (1980) Thresholds for object motion perception raised by concurrent head movements. Pflugers Arch 384(Suppl R33):131

Duersteler MR (2005) Asymmetries in surround and central motion perception for rotating patterns. Abstract for the Meeting of the Society for Neuroscience, USA. Program no 390, 18, 2005; Viewer/itinerary Planner, Washington, DC; Society for Neuroscience

Durgin FH, Gigone K, Scott R (2005) The perception of visual speed while moving. J Exp Psychol Hum Percept Perform 31(2):339-353

Mesland BS, Wertheim AH (1996) A puzzling percept of stimulus stabilization. Vis Res 36(20):3325-3328

Mittelstaedt H (1990) Basic solutions to the problem of head-centric visual localization. In: Warren R, Wertheim AH (eds) Perception and control of self-motion. Lawrence Earlbaum, Hillsdale NJ, pp 257-287

Murphy BJ (1978) Pattern thresholds for moving and stationary gratings during smooth pursuit eye movements. Vis Res 18:521530

Nakayama K (1981) Differential motion hyperacuity under conditions of common image motion. Vis Res 21:1475-1482

Pavard B, Berthoz A (1977) Linear acceleration modifies the perceived velocity of a moving visual scene. Perception 6:529 540

Pelah A, Thurell AEI (2001) Reduction of perceived visual speed during locomotion: evidence for quadrupedal perceptual pathways in human? J Vis I:307a. http://www.journalofvision.org/1/ $3 / 307$

Probst T, Brandt T, Degner D (1986) Object motion-detection affected by concurrent self-motion perception. Psychophysics of a new phenomenon. Behav Brain Res 22:1-11

Thurell AEI, Pelah A (2002) Reduction of perceived visual speed during walking: effect dependent upon stimulus similarity to the visual consequences of locomotion. J Vis 2:628a. http:// www.journalofvision.org/2/7/628

Von Holst E, Mittelstaedt H (1950) Das Reafferenzprinzip (Wechselwirkung zwischen Zentralnervensystem und Peripherie) Naturwissenschaften 37:460-476

Wallach H (1987) Perceiving a stable environment when one moves. Annu Rev Psychol 38:1-29

Wertheim AH (1981) On the relativity of perceived motion. Acta Psychol (Special issue on motion perception) 48:97-110

Wertheim AH (1994) Motion perception during self-motion: the direct versus inferential controversy revisited. Behav Brain Sci 17(2):293-355

Wertheim AH, Niessen MW (1986) The perception of relative motion between objects during pursuit eye movements. Perception 15(1):A9

Wertheim AH, Van Gelder P (1990) An acceleration illusion caused by underestimation of stimulus velocity during pursuit eye movements: the Aubert-Fleischl phenomenon revisited. Perception 19(4):471-482 [Erratum in: Perception 19(5), pp 700] 